\title{
ÉGYPTE PHARAONIQUE
}

\section{Erkundungen zur Reihenfolge der Zeichen im ägyptologischen Transkriptionsalphabet}

\section{Das geltende ägyptologische Transkriptionsalphabet: ein Kuriosum}

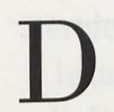

IE Reihenfolge der Zeichen im ägyptologischen Transkriptionsalphabet wird heute standardmäßig, wie folgt, angenommen (Transkriptionszeichen nach "Wörterbuch der ägyptischen Sprache», nach dem Schrägstrich gängige Alternativnotationen; vgl. auch Abb. 1) (1):

3, $i / j, j / y / j j, r, w, b, p, f, m, n, r, h, h, \underline{h}, \underline{h}, s / z, \dot{s} / s, \dot{s}, \underline{k} / q, k, g, t, \underline{t}, d, \underline{d}$

Üblich sind gewisse Vereinfachungen bzw. Erweiterungen, die sich aus Veränderungen des Phonembestandim Laufe der ägyptischen Sprachgeschichte ergeben. So werden erstens $s / z$ und $s / s$, Zeichen für zwei Phoneme, die nach dem Alten Reich (oder in dessen Ende) infolge eines Lautwandels $s / z>s / s$ zusammenfielen, vielfach als ein einziges Zeichen, $s$, behandelt $\left({ }^{2}\right)$. Zweitens wird $j / y / j j$, zum mindesten das anlautende, auch wenn als $j$ oder $y$ von $i$ oder als $j j$ von $j$ unterschieden, gerne unter $i / j$ eingeordnet, da $j / y / j j$ als Phonem überhaupt erst nach dem Alten Reich (oder in dessen Ende) sicher faßbar ist (als $j / y<w$ ) und im Anlaut erst in der Schreibung kanaanäischer Fremdwörter im Neuen Reich geläufig wird $\left({ }^{3}\right)$. Die genannten Modifikationsmöglichkeiten und etwaige andere, nicht genannte, sind für die vorliegende Untersuchung ohne besonderen Belang. Ebensowenig kommen die speziellen Adaptationen in Betracht, die man bei der Anwendung des Transkriptionsalphabets für das Demotische vorzunehmen hat (unter denen die Einfügung des Zeichens $l$ hinter dem Zeichen $r$ die wichtigste ist) $\left(^{4}\right)$.

(1) Als maßgebende Werke für die alphabetische Folge sind zu betrachten: A. Erman / H. Grapow (Hg.), Wörterbuch der ägyptischen Sprache, Leipzig bzw. Berlin, 1926-1963 ; A. H. Gardiner, Egyptian Grammar, ${ }^{3}$ London, 1957, S. 27 und 549-604.

(2) So Gardiner, op. cit.; anders Erman/Grapow, op. cit.

(3) So Erman/Grapow, op. cit.; anders Gardiner, op. cit.

(4) Siehe etwa W. Erichsen, Demotisches Glossar, Kopenhagen 1954. 


\begin{tabular}{|c|c|c|c|}
\hline $\begin{array}{l}\text { Laufende } \\
\text { Nummer }\end{array}$ & $\begin{array}{l}\text { Repräsentatives } \\
\text { Einkonsonanten- } \\
\text { zeichen }\end{array}$ & $\begin{array}{l}\text { Übliches } \\
\text { Transkriptions- } \\
\text { zeichen }\end{array}$ & $\begin{array}{l}\text { Lautphysiologische } \\
\text { Gruppe }\end{array}$ \\
\hline $\begin{array}{l}1 \\
2 \\
3 \\
4 \\
5\end{array}$ & $\frac{1}{8}$ & $\begin{array}{l}3 \\
i / j \\
j / y / j j \\
c \\
w\end{array}$ & $\begin{array}{l}\text { ehemals als Vokale } \\
\text { aufgefaßt }\end{array}$ \\
\hline $\begin{array}{l}6 \\
7\end{array}$ & $\begin{array}{l}\perp \\
\square\end{array}$ & $\begin{array}{l}b \\
p\end{array}$ & labiale Explosive \\
\hline 8 & $\infty$ & $f$ & labiale Frikativa \\
\hline $\begin{array}{r}9 \\
10 \\
11\end{array}$ & 0 & $\begin{array}{l}m \\
n \\
r\end{array}$ & Liquide \\
\hline $\begin{array}{l}12 \\
13 \\
14 \\
15\end{array}$ & $\begin{array}{l}10 \\
8 \\
0 \\
\infty\end{array}$ & $\begin{array}{l}h \\
h \\
h \\
h \\
\underline{h}\end{array}$ & gutturale Frikative \\
\hline $\begin{array}{l}16 \\
17 \\
18\end{array}$ & $\prod_{\infty}^{\infty}$ & $\begin{array}{l}s / z \\
\dot{s} / s \\
\dot{s}\end{array}$ & dentale Frikative \\
\hline $\begin{array}{l}19 \\
20 \\
21\end{array}$ & $\begin{array}{l}\Delta \\
\infty\end{array}$ & $\begin{array}{l}k / q \\
k \\
g\end{array}$ & gutturale Explosive \\
\hline $\begin{array}{l}22 \\
23 \\
24 \\
25\end{array}$ & $\begin{array}{l}0 \\
= \\
=\end{array}$ & $\begin{array}{l}t \\
\underline{t} \\
d \\
\underline{d}\end{array}$ & $\begin{array}{l}\text { dentale Explosive und Ver- } \\
\text { wandte }\end{array}$ \\
\hline
\end{tabular}

Авв. 1. - Das geltende ägyptologische Transkriptionsalphabet 
Die Reihenfolge der Transkriptionszeichen gilt, aus heutiger Perspektive, schon immer, solange nämlich schon, wie die kollektive Erinnerung der heutigen ägyptischen Sprachwissenschaft zurückreicht. Sie galt bereits in den achtziger Jahren des vorigen Jahrhunderts, als Adolf Erman seine ersten grundlegenden Arbeiten schrieb. Erman übernahm die Reihenfolge fertig als Erbstück. Da abər weder Erman selbst noch irgendein anderer Gefolgsmann aus Ermans "Berliner Schule» Anlaß fand, die Frage der Reihenfolge noch einmal zu erörtern, hat sich ihre Herkunft und ihre Begründung verflüchtigt — wie überhaupt durch Ermans Neubeginn die Verbindungslinien zur älteren Ägyptologie im Bereich der Sprachforschung weitgeh'end abgeschnitten wurden.

Immerhin kann man Sinn und Zweck der Anordnung ohne wissenschaftsgeschichtliche Recherchen auch heute noch zu einem guten Teil feststellen: Man kann sie an Eigenheiten des Alphabets selbst ablesen. So läßt sich erkennen (vgl. Abb. 1), daß - hier und im folgenden stets die traditionelle Terminologie — «labiale Explosive» $(b, p)$, «dentale Explosive" und deren - wie man informell sagen könnte - Verwandte $(t, \underline{t}, d, \underline{d})$ "gutturale Explosive » $(k / q, k, g)$ sowie «Liquide» $(m, n, r)$ und «Frikative» ("gutturale» $h, \underline{h}, \underline{h}, \underline{h}$; «dentale» $s / z, s / s, s$ ) jeweils Gruppen bilden. Es läßt sich aber nicht erklären, warum in der Gruppe der «Labiale» die "Lenis» $b$ vor der "Fortis" $p$ steht, in der Gruppe der «Gutturale» dagegen die "Lenis» g nach der "Fortis" $k$ (u. dgl. m.), warum $f$ nach $b$ und $p$, d.h. bei den "Labialen ", steht, nicht bei den übrigen "Frikativen ", oder — ein besonders merkwürdiger Befund warum die Gruppen der "Liquide» und "Frikative» die "Explosive " in zwei Sequenzen aufsprengen, die "labialen Explosive», die vor den "Liquiden " und "Frikativen " stehen, und die "gutturalen " und "dentalen" "Explosive», die hinter den "Liquiden " und "Frikativen " stehen, bzw. anders herum betrachtet: warum die "Explosive» nicht in einer einzigen Sequenz zusammengefaßt sind. Als - unter anderem Gesichtspunkt - sinnreich läßt sich verstehen, daß $\underline{t}$ auf $t$ folgt und $\underline{d}$ auf $d$ : weil die Reihenfolge nahe beieinanderläßt, was durch den numerisch bedeutsamen Lautwandel $\underline{t}>t \mathrm{bzw} . \underline{d}>d$ historisch eng verbunden ist (so wie auch $s / z$ und $s / s$ beieinanderstehen, die über den totalen Lautwandel $s / z$ $>\dot{s} / s$ historisch verbunden sind).

Noch am besten präsent ist heute die Ratio für die Anfangssequenz des Alphabets mit der kuriosen Folge $3, i / j, j / y / j j, r, w$. Es handelt sich hier um die Zeichen für diejenigen Phoneme, die man in der Frühzeit der Ägyptologie allgemein für Vokale hielt und später außerhalb der 
«Berliner Schule» als solche erachtete. Der Sachverhalt ist einfach deshalb präsent, weil die Kontroverse um den Status der Phoneme - hie "Vokal", hie "Konsonant" — noch zur Zeit der "Berliner Schule" lebendig war.

Die Beobachtungen und Fragen ließen sich vermehren, die Gesichtspunkte der Zerlegung in Teilsequenzen ließen sich anders formulieren es wurden hier im Vorgriff bereits die wissenschaftsgeschichtlich obwaltenden gewählt —, wie man es auch dreht und wendet: Die Reihenfolge der Zeichen zeigt Sinnreiches und Unerklärliches in bunter Mischung. Wirklich verstehbar wird der Befund nur aus seiner wissenschaftsgeschichtlichen Genese. Hierzu das folgende.

\section{Lautwerte hieroglyphischer Schriftzeichen als Äquivalente zu Lautwer-} ten koptischer Buchstaben: das koptische Alphabet

\subsection{Vorstufe: das griechische Alphabet}

Der erste konsolidierte Schritt zur Wiedergewinnung der altägyptischen Sprache war die Bestimmung hieroglyphischer Äquivalente zu griechischen und - weniger wichtig - lateinischen Buchstaben in hieroglyphischen Transkriptionen von Namen und Titeln des ptolemäischen Königshauses und römischer Kaiser durch Jean François Champollion, der 1822 in seinem «Lettre à M. Dacier» eine stringente Lösung des Problems vorlegte. Es lag auf der Hand, die Korrespondenztabelle der Schriftzeichen nach dem griechischen Alphabet zu ordnen, da für die griechische Seite der Äquivalenzrelationen ein traditionelles Ordnungsprinzip verfügbar war, nicht jedoch für die ägyptische $\left(^{1}\right)$. Die Orientierung am griechischen Alphabet geht so weit, daß selbst für zweilautige Zeichen des griechischen Alphabets wie $\xi$ und $\psi$ schematisch hieroglyphische Äquivalente angegeben werden, obwohl sich diese Äquivalente deutlich aus den entsprechenden Einzellauten zusammensetzen $(\xi \hat{=} k+s, \psi \hat{=} p+s)$. Umgekehrt ist an das Ende des griechischen Alphabets die Lautfolge $\tau o$ bzw. $\delta o$ als eine Art "Zusatzbuchstaben » angehängt, weil dieser auf der hieroglyphischen Seite ein einziges Zeichen gegenübersteht, $=\left(t_{3}\right)$, von Champollion verstanden als to, do. Als

(1) J. F. Champollion, Lettre à M. Dacier ... relative à l'alphabet des hiéroglyphiques phonétiques employés par les égyptiens pour inscrire sur leurs monuments les titres, les noms et les surnoms des souverains grecs et romains, Paris, 1822, Taf. VI. 
kleine Beckmesserei : Im griechischen Alphabet ist die Reihenfolge $\eta-\theta$ in $\theta-\eta$ umgekehrt worden.

\subsection{Koptisches oder hebräisches Alphabet}

Der Durchbruch bei der Wiedergewinnung der altägyptischen Sprache gelang Champollion durch die Bestimmung von Elementen der koptischen Sprache (Wörter, grammatische Formen) als Äquivalente hieroglyphisch-ägyptischer. Das Denkmal dieses Durchbruchs ist sein "Précis du système hiéroglyphique" aus dem Jahre 1824.

Hier würde man eigentlich eine Anordnung der hieroglyphischen Lautzeichen nach dem koptischen, d.h'. dem um Zusatzzeichen vermehrten griechischen Alphabet erwarten. Tatsächlich wurde - mit Modifikationen, auf die noch die Rede kommen wird (s. unten $\S 2.3$ ) das koptische Alphabet benutzt, daneben aber auch das hebräische $\left.{ }^{(}\right)$. Neben den koptischen (und den zuvor schon entdeckten griechischen, s. oben § 2.1) Äquivalenten werden nämlich auch hebräische gegeben. Letztere, an sich von geringerem Gewicht, haben dann aber zum mindesten mit den Ausschlag gegeben, in der abschließenden Liste der Lautzeichen diese nach dem hebräischen Alphabet zu ordnen (ein anderer Grund für die Entscheidung mag darin liegen, daß ein tieferer Zusammenhang des Ägyptischen mit dem altehrwürdigen Hebräisch erahnt bzw. gesucht wurde). Der Buchstabe $\ddot{w}(\dot{s})$ wird übergangen, weil dessen Äquivalente bereits unter $\checkmark(s)$ günstig untergebracht werden konnten, somit ש ohne diakritischen Punkt für ש $(\tilde{s})$ stehen konnte. Es mußten aber auch hier wieder, wie beim oben $\S 2.1$ behandelten griechischen Alphabet, "Zusatzbuchstaben » angehängt werden für Zeichen, für die das hebräische Alphabet keine Äquivalente bot. So wurden angehängt in dieser Reinenfolge : die beiden koptischen "Zusatzbuchstaben » q $(f)$ und $\boldsymbol{\dagger}(t i)$; die koptischen/griechischen Vokale $o(o), \omega(\bar{o}), \iota(i) / \eta$ $(\bar{e}$, d.i. $\bar{\imath}) / \varepsilon \iota(e i$, d.i. $\bar{\imath}) /(\alpha \iota$, d.i. $e)$; die Doppellaute $\xi(k s)$ und $\psi(p s)$ des griechischen Alphabets (mit denen zusammen eigentlich auch das schon vorher eingeordnete koptische † (ti) hätte stehen können); sowie einige Zeichen mit Doppellauten des Hieroglyphischen, für die keines der in Frage kommenden Alphabete ein Äquivalent bot: $m a$ (d.i. $m r), m n$ (d.i. $m n)$ und to bzw. tō (d.i. $t_{3}$ ).

(1) J. F. Champollion, Précis du système hiéroglyphique des anciens égyptiens, Paris, 1824 (zitiert nach der 2. Aufl. von 1827-28), S. 360 (koptisches Alphabet); Taf. A-K (hebräisches Alphabet). 
Nur das koptische Alphabet, nicht also das griechische und nicht das hebräische, bot für alle von Champollion bestimmten hieroglyphischen Laute ein Äquivalent - abgesehen natürlich von einigen «Doppellauten », für die keines der Alphabete ein solches bot. Hinzu kommt, daß die Sprache der hieroglyphischen Texte als Koptisch identifiziert worden war. So ist es denn auch nicht zu verwundern, daß Champollions definitive Lösung, wie sie in seiner posthumen, 1836 erschienen "Grammaire égyptienne » vorliegt, - mit noch zu besprechenden Modifikationen (s. unten $\S 2.3$ ) - die Anordnung nach dem koptischen Alphabet ist (1). (Zum später erneuerten Interesse am hebräischen Alphabet s. unten $\S 3$.)

\subsection{Reduktion des koptischen Alphabets}

Die Zuordnungen zwischen hieroglyphischen Zeichen und koptischen Buchstaben bzw. Buchstabenfolgen sind in der Regel sowohl in der einen wie in der anderen Richtung mehrdeutig. Z.B. ist koptisches $\mathbf{M}(m)$ sowohl ein Äquivalent für hieroglyphisches $(m)$ als auch für hieroglyphisches $\sqsubset(m)$, umgekehrt ist ein hieroglyphisches $\checkmark(k)$ sowohl ein Äquivalent für koptisches $\Gamma(g)$ wie für koptisches $\kappa(k)$. Erstere Diskrepanz, die Frage der hieroglyphischen Allographen, ist kein Problem der alphabetischen Anordnung, insofern also hier nicht weiter zu behandeln; gleichwohl spielt die Frage in die Anordnung des sprachlichen Materials hinein, wie ein Blick in das «Wörterbuch der ägyptischen Sprache " zeigt, wo bei der Feinsortierung des Materials Homonyme u.a. nach Homographen sortiert werden, z.B. stehen unter Wörtern mit der Konsonantenfolge $m r$ zuerst diejenigen, die ohne Zweikonsonantenzeichen $m r$ geschrieben werden, dann die mit Zweikonsonantenzeichen ? $(m r)$, dann die mit Zweikonsonantenzeichen $\leftarrow(m r)$, dann die mit Zweikonsonantenzeichen $\simeq(m r)\left({ }^{2}\right)$. Das Alphabet selbst dagegen ist betroffen durch Mehrdeutigkeiten der zweiten Art. Sie haben bereits Champollion dazu veranlaßt, die hieroglyphischen Äquivalente von $\mathrm{\kappa}(k)$ und $6\left(k^{\prime}\right)$ zusammenzufassen und alphabetisch an der Stelle des ersten der " $k$-Laute», $\mathbf{r}(g)$, einzuordnen, desgleichen diejenigen von $\mathbf{T}(t)$ und $\boldsymbol{\Theta}\left(t^{h}\right)$ analog bei $\delta(d)$, diejenigen von $\pi(p)$ und $\phi\left(p^{h}\right)$ bei $\Pi(p)$, sowie die Vokale (des Koptischen), bei denen Mehrdeutigkeiten herüber und

(1) J. F. Champollion, Grammaire égyptienne, Paris, 1836, S. 34.

(2) Erman/Grapow, op. cit., II, S. 94-98. 
hinüber kulminieren, überhaupt aus dem Alphabet herauszunehmen und als besondere Gruppe an dessen Spitze zu stellen $\left(^{1}\right)$.

Auf solcher Basis, unter Zugrundelegung der Lautwerte, die die Hieroglyphen in griechisch-römischer Zeit besaßen, hat dann Richard Lepsius 1837 abschließend ein Alphabet festgelegt, das aus einer Gruppe von drei Vokalen plus einer Gruppe von zwölf Konsonanten besteht, innerhalb deren, unter Übergehung der nicht benötigten Buchstaben, die Reihenfolge des koptischen Alphabets gilt $\left({ }^{2}\right)$ :

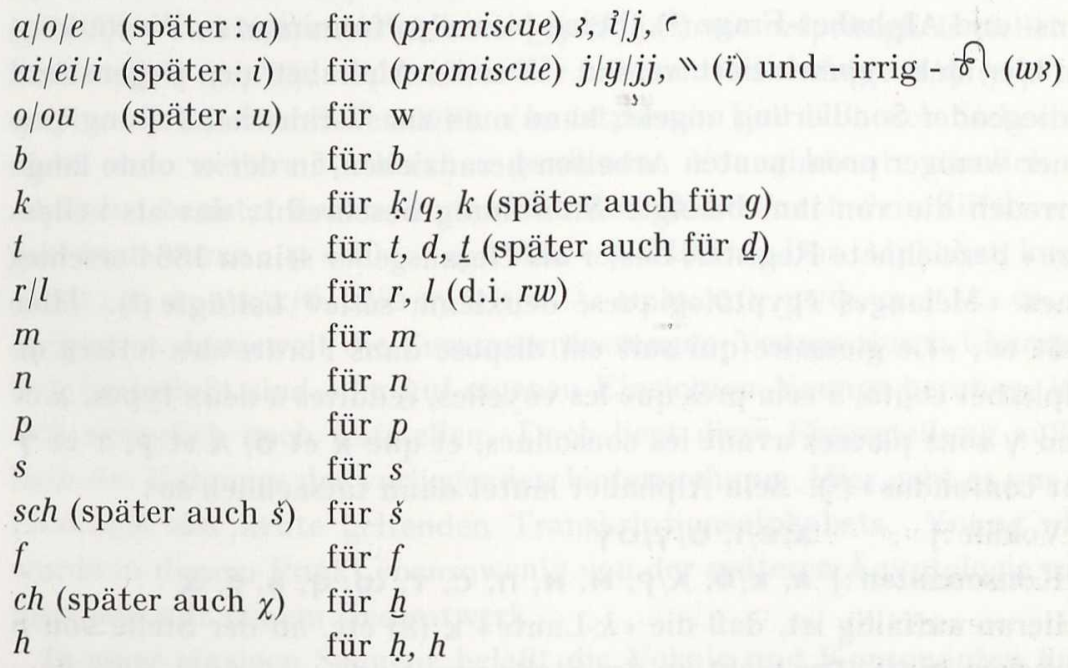

Für lexikographische Zwecke haben weder Champollion noch Lepsius das koptische Alphabet genutzt : Lepsius, weil er der Lexikographie fern stand, Champollion, weil er sein — posthum 1841 erschienenes — «Dictionnaire égyptien " nach anderem Prinzip aufbaute : dem Vorbild chinesischer Wörterbücher folgend, nach dem Bildgehalt der Schriftzeichen, d.h. auf der Basis der hieroglyphischen Zeichenliste $\left(^{3}\right)$.

Ein modifiziertes koptisches Alphabet angewandt hat dagegen vor allem François Joseph Chabas, seines Zeichens Weinhändler in Chalon-

(1) Champollion, Précis, S. 360f.; id., Grammaire, S. 35-46.

(2) R. Lepsius, Lettre à $M$. le professeur H. Rosellini ... sur l'alphabet hiéroglyphique, Rome, 1837, Taf. A.; zu den in Klammern angegebenen späteren Transkriptionszeichen s. id., Über eine hieroglyphische Inschrift am Tempel von Edfu..., in APAW phil.-hist., 1856, S. 69-114, bes. S. 112f. ; sowie id., Königsbuch der alten Ägypter, Berlin, 1858, S. 169f. (Anzahl und Identität der Zeichen sind in diesen Arbeiten immer noch die von 1837, die Reihenfolge ist dagegen nicht mehr die des koptischen Alphabets).

(3) J. F. Champollion, Dictionnaire égyptien en écriture hiéroglyphique, Paris, 1841. 
sur-Saône, einer der Großen der Ägyptologie des 19. Jh., der in keiner Geschichte der Ägyptologie fehlen darf. Er trat für dieses Alphabet noch zu einer Zeit ein, als die letztlich siegreichen Berliner Kollegen die Weichen längst in andere Richtung gestellt hatten, und — «au nom de l'école de Champollion» $\left.{ }^{1}\right)$ — mit solcher Hartnäckigkeit, daß ihn Georg Ebers, gewiß kein Doktrinär in Sachen Transkription, in einem Brief von 1874 den "Robinson de l'île de la transcription copte» nannte $\left({ }^{2}\right)$. Chabas äußerte sich mehrfach ausführlich zur Transkriptions- und Alphabet-Frage $\left(^{3}\right)$. Seine bisweilen fulminanten Worte können hier nicht reproduziert werden. Was das Alphabet, den Gegenstand vorliegender Sondierung angeht, kann man zur Veranschaulichung eine seiner weniger prominenten Arbeiten heranziehen, in der er ohne lange Vorreden die von ihm befolgte Anordnung beschreibt: das als «Glossaire» bezeichnete Register, das er als Herausgeber seinen 1864 erschienenen "Mélanges égyptologiques, deuxième série» beifügte $\left(^{4}\right)$. Hier heißt es : "Le glossaire qui suit est disposé dans l'ordre des lettres de l'alphabet copte, à cela près que les voyelles, réduites à deux types, a et o ou $\boldsymbol{Y}$ sont placées avant les consonnes, et que $\boldsymbol{\kappa}$ et $\boldsymbol{\sigma}, \boldsymbol{\lambda}$ et $\mathbf{P}, \mathbf{T}$ et $\boldsymbol{\top}$ sont confondus» $\left(^{5}\right)$. Sein Alphabet lautet dann tatsächlich so :

- [Vokale:] a/€/I, O/Y/OY

- [Konsonanten:] B, K/6, $\lambda / \mathrm{P}, \mathrm{M}, \mathrm{N}, \mathrm{\Pi}, \mathrm{C}, \mathrm{T}, \boldsymbol{c}, \mathrm{q}, \boldsymbol{b}, \mathbf{2}, \mathbf{x}$

Hieran auffällig ist, daß die " $k$-Laute» $\boldsymbol{\kappa}(k)$ etc. an der Stelle von $\mathbf{r}$ $(g)$ stehen, $\mathbf{T}(t)$ aber nicht an der Stelle von $\boldsymbol{\lambda}(d)$.

Eine andere zeitgleiche, jedoch etwas chaotische Adaption des koptischen Alphabets durch Heinrich Brugsch wird unten ( 5 5.3) zur Sprache kommen.

(1) F. Chabas, Voyage d'un égyptien en Syrie, en Phénicie, en Palestine, \&c. au $X I V^{\text {me }}$ siècle avant notre ère, Chalon-sur-Saône - Paris, 1866, S. VII.

(2) F. Chabas, Euvres diverses, Bd. 1, S. CXXXV, Anm. 2 (es ist der Zitatstelle nicht zu entnehmen, ob die Charakterisierung von Chabas ursprünglich französisch formuliert war oder erst von dessen Biographen ins Französische übersetzt wurde).

(3) S. vor allem: F. Chabas, Le papyrus magique Harris, Chalon-sur-Saône, 1860, S. 199-202; id., Voyage, S. 1-6; 13-18; 353 ; ferner : id., Les papyrus de Berlin, récits d'il y a 4000 ans, Chalon-sur-Saône, 1863 (= Euvres diverses, Bd. 2, hiernach zitiert), S. 347, sowie die in der folgenden Anm. zitierte Arbeit. - S. auch F. Chabas, Euvres diverses, Bd. 1, S. LIII (Brief an Lepsius vom 7.1.1864); XLIX (Brief vom 22.5.1863).

(4) F. Chabas (Hg.), Mélanges égyptologiques, $2^{\mathrm{e}}$ série, Chalon-sur-Saône, 1864, S. 307-323.

(5) Chabas, Mélanges, $2^{\mathrm{e}}$ série, S. 307. 
Anmerkung :

Angewandt hat ein modifiziertes koptisches Alphabet auch Thomas Young (†1829) in seinen posthum 1830 erschienenen «Rudiments of an Egyptian Dictionary » $\left(^{1}\right)$. Der Haupt-Unterschied zwischen seinem Alphabet und dem koptischen Alphabet der Linie Champollion/Lepsius/spätere Ägyptologie liegt darin, daß er Vokale und Konsonanten in einer einzigen Sequenz beläßt. Bei den Vokalen faßt er $\mathbf{\epsilon} / \mathbf{1} / \mathbf{H}(e / i / \bar{e})$ zusammen sowie $\mathbf{o} / \mathbf{Y}(o / u)$, bei den Konsonanten außer $\mathbf{r} / \mathbf{k} / \mathbf{x} / \mathbf{6}$ $\left(g / k / k^{h} / k^{\prime}\right)$ und $\boldsymbol{\lambda} / \boldsymbol{\theta} / \mathbf{T}\left(d / t^{h} / t\right)$ auch $2 / \boldsymbol{b}(h / h)$ sowie ursprünglich vielleicht auch $\Pi / \phi / \varphi\left(p / p^{h} / f\right)$, wie sich aus der falschen Reihenfolge $\pi / \phi\left(p / p^{h}\right)$ $q(f)-P(r)$ erschließen läßt. (In letzterem Fall läßt sich Sicherheit nicht ohne weitere Recherchen gewinnen. Young könnte nämlich ein Alphabet benutzt haben, in dem die Buchstaben nach dem Ziffernwert

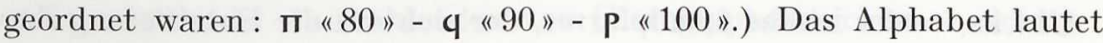

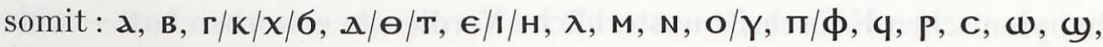
$x, 2 / b$. - Inwieweit die Zusammenfassungen Youngs durch Champollion beeinflußt sind oder auf eigenen Einsichten Youngs beruhen, läßt sich sicherlich noch feststellen. Doch liegt diese Fragestellung außerhalb des Rahmens der vorliegenden Untersuchung. Hier geht es um die Herkunft des heute geltenden Transkriptionsalphabets. Young aber wurde in diesem Punkt ebensowenig von der späteren Ägyptologie rezipiert wie mit seinem Gesamtwerk.

In einer einzigen Sequenz beläßt die Vokale und Konsonanten auch Samuel Sharpe in seinen 1837 erschienenen "Rudiments of a Vocabulary of Egyptian Hieroglyphics». In der unter dem Titel «Egyptian Hieroglyphics...» erschienenen 2. Auflage von 1861 übernimmt er dann die inzwischen üblich gewordene Aufteilung in Vokale und Konsonanten.

§3. Lautwerte hieroglyphischer Schriftzeichen als Äquivalente zu Lautwerten hebräischer Buchstaben: das hebräische Alphabet

Einen entschiedenen Schritt weg von der griechisch-römischen bzw. koptischen Zeit zurück in die altägyptische und zugleich weg vom koptischen oder gar griechischen Lautstand in Richtung auf das urver-

(1) T. Young, Rudiments of an Egyptian Dictionary in the Ancient Enchorial Character ..., London, 1830, bes. die Zusammenstellung S. 39. 
wandte Semitisch taten Edward Hincks und, teilweise unabhängig, teilweise letzterem folgend, Heinrich Brugsch. Sie bestimmten Äquivalenzen zwischen Lauten des Hieroglyphisch-Ägyptischen und des Semitischen, d.h. des Hebräischen, anhand vor allem der kanaanäischen Namen und Fremdwörter in den Texten der früheren Ramessidenzeit (bei Hincks ist dies "the age of the papyri», die Zeit von Ramses I. bis Ramses IV. oder V.). Daneben spielen bei der Bestimmung der ägyptischen Lautwerte eine Rolle: die Wiedergabe ägyptischer Wörter in hebräischer oder griechischer Schrift; Beobachtungen über die NichtAustauschbarkeit von Schriftzeichen in älteren Hieroglyphentexten, die in griechisch-römischer Zeit promiscue gebraucht werden; sowie der Vergleich von altägyptischen Wörtern, die mutmaßlich mit solchen anderer Sprachen genetisch verwandt sind.

Hincks - der irische Landpfarrer, der sich um die Entzifferung der babylonischen Keilschrift unsterbliche Verdienste erworben hat - widmete der Frage der Lautwerte eine gründliche und umsichtige Darstellung, die 1847 als Abhandlung ("Transaction») der Royal Irish Academy erschien ( $\left.{ }^{1}\right)$. Er setzt bei dem Problem der Interpretation der heute so genannten - Syllabischen Schrift an, in der die in Rede stehenden kanaanäischen Namen und Fremdwörter geschrieben sind. Sehr richtig sieht er, daß in diesen Schreibungen den Konsonanten, die in den semitischen, d.h. hebräischen, Lautformen Äquivalente besitzen, oft Schriftzeichen folgen, für die es in den semitischen Lautformen keine Entsprechungen gibt - aus heutiger Sicht die nicht-konsonantische Komponente der Syllabischen Schrift. Hincks meint, es handle sich bei diesen Zusätzen ohne semitische Entsprechung um "expletive characters ", d.h., ergänzende Buchstaben, die den vorangehenden konsonantischen Buchstaben zu einem Buchstaben-Namen ergänzen $\left({ }^{2}\right)$. Man könne also im Ägyptischen statt mit Buchstaben auch mit Buchstaben-Namen schreiben, in der Art etwa, als wenn man im Englischen statt «Rome» auch "Ar-o-em-e», die Folge der Buchstaben-Namen für

(1) E. Hincks, An Attempt to Ascertain the Number, Names, and Powers of the Letters of the Hieroglyphic, or Ancient Egyptian Alphabet, Grounded on the Establishment of a New Principle in the Use of Phonetic Characters, in Royal Irish Academy, Transactions 21 (1848), S. 132-232 (zum tatsächlichen Erscheinungsjahr $1847 \mathrm{~s}$. K. H. Cathcart / P. Donlon, Edward Hincks (1792-1866): A Bibliography of his Publications, in Orientalia 52 (1983), S. 325-356, bes. S. 335 und allgemein S. 328 ; die auf S. 335 gegebene Inhaltsangabe zu diesem Werk ist unzutreffend).

(2) Hincks, op. cit., S. 139-161. 
《r», " 0 », " $\mathrm{m} »$, , $\mathrm{e} »$, schreiben könnte $\left({ }^{1}\right)$. Diese Erklärung ist, wie man heute weiß, falsch. Sie muß aber deshalb erwähnt werden, weil Richard Lepsius daraus Schlüsse für die Transkription des Ägyptischen gezogen zu haben scheint - andere, aber ebenso unzutreffende - , auf die unten ( 5 5.2) die Rede zu bringen sein wird. Ansonsten hätte man diesen Punkt ohne weiteres übergehen dürfen, denn, anders als Hincks selbst dachte, bildet seine Interpretation der Syllabischen Schrift keineswegs die unabdingbare Voraussetzung für seine anschließenden Lautwertbestimmungen. Einzige Voraussetzung ist, daß man die Zeichen, die Hincks als "expletive characters» eliminiert, auf irgendeine Weise aus der Konsonantenfolge ausblendet, siesauf irgendeine Weise «überliest». Dies tut man heute anders, als Hincks es sich zurechtlegte, aber seine Lösung war durchaus zweckdienlich, letztlich sogar korrekt, weil der Grundgedanke - Ausblenden von Hieroglyphenzeichen — richtig ist.

Nun also zur Lautwert-Bestimmung: Hincks weist nach $\left({ }^{2}\right)$, daß in der früheren Ramessidenzeit (in Klammern die entsprechenden heutigen Transkriptionszeichen)

$\left.{ }^{c}\right), 4(i / j)$ und $(3)$, die man bis dahin als Allographen für $a$ betrachtet hatte, verschiedene Lautwerte besaßen,

$-\underset{\sim}{-c}\left({ }^{c}\right)$ den Lautwert $y\left({ }^{c}\right)$,

- $4(i / j)$ den Lautwert $\boldsymbol{\aleph}($ (') besaß,

so daß als a nur (3) übrig bleibt, für dessen Lautwert das ihm bekannte Material keine Anhaltspunkte bot (auch heute noch kann man den Lautwert nicht mit ramessidenzeitlichem Material bestimmen). Er sucht, da er keine letzte Sicherheit gewinnt, wenigstens plausibel zu machen, daß

$-\Delta(k / q)$ verschieden ist von $k$ und den Lautwert $p(q)$ besaß.

Er sieht, daß

$-\subseteq(d$, d.i. $t)$ verschieden ist von $d$, und erwägt, ob es den Lautwert v

(t) besessen haben könnte, verwirft diese Lösung aber wieder in einer nachgetragenen Fußnote, leider ohne Angabe von Gründen.

Schließlich weist er nach, daß

$-2(\underline{d}$, d.i. $\underline{c})$ (und entsprechend $\underline{d}\left(\underline{d} 3\right.$, d.i. $\left.\underline{c}_{3}\right)$ ) verschieden ist von $t$ und

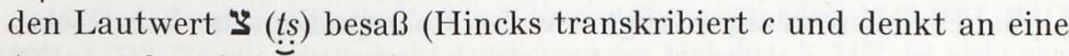
Aussprache wie $\underline{t s}$ oder $t s$ ).

(1) Hincks, op. cit., S. 147.

(2) Hincks, op. cit., S. 161-232. 
Weniger glücklich ist er bei der Bestimmung der Lautwerte von $\mathbb{d}(b)$ und $f\left(w_{3}\right)$, die er für gleich hält $(u, w, v$, alt etwa bilabiales $w)$ sowie von $\curvearrowleft(f)$, dessen Lautwert er eher bei $w, v$ suchen möchte (andere zeittypische - Irrtümer bei der Lautwertbestimmung - u.a. die Auffassung einer Reihe von Zweikonsonantenzeichen als Einkonsonantenzeichen - können hier übergangen werden, da sie auf Menge und Art der Lautwerte und somit auf den Umfang des Alphabets keinen Einfluß haben). Ansonsten bleibt Hincks bei den Lautwerten, wie sie damals in der Ägyptologie rezipiert waren, d.h. bei den Lautwerten, wie sie Christian Carl Josias Bunsen festgelegt hatte, d.h. letztlich : bei den Lautwerten von Richard Lepsius (zu Bunsen/Lepsius s. unten §4).

Die entscheidenden «semitischen» Einsichten von Hincks, um diese der Klarheit wegen noch einmal zu resümieren, sind:

$$
\begin{aligned}
& -4(i / j) \triangleq \aleph(') \\
& \longrightarrow\left({ }^{c}\right) \triangleq \\
& -\Delta(k / q) \triangleq P(q) \\
& -\Longrightarrow(d \text {, d.i. } t) \hat{\neq} d \text {, } \triangleq t \text { (?; verworfen) }
\end{aligned}
$$

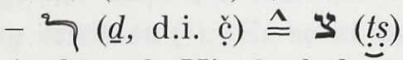

Anders als Hincks behändelt Brugsch die Frage rasch und eher beiläufig $\left(^{1}\right)$. Brugsch stimmt, was ${ }^{c}, i / j$ und $k / q$ angeht, mit Hincks überein. Seine Behandlung der «Dentale » zeigt elementare Mängel $(\bullet(t) \hat{=} \bullet(t)$, $\checkmark(\underline{d}$, d.i. $\grave{c}) \hat{f}(\underline{d}(3)$, d.i. $\grave{c}(3)))$, die der Lösung der eigentlichen Problemfälle, die Hincks sehr richtig erkannt hatte, im Wege stehen. Dage-

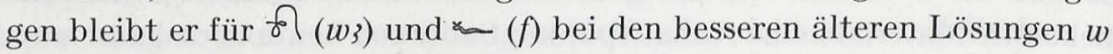
bzw. $f$. Beachtenswert ist der Vorschlag 屯 $(g)$ als $g$ zu lesen (ein Vorschlag, über den bis zum heutigen Tag das letzte Wort nicht gesprochen ist).

Brugsch faßt seine Ergebnisse in einem «ägyptisch-semitischen Alphabet" zusammen, einem hebräischen Alphabet, das durch Zerlegung

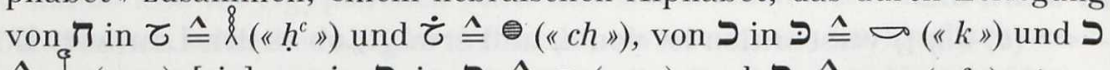

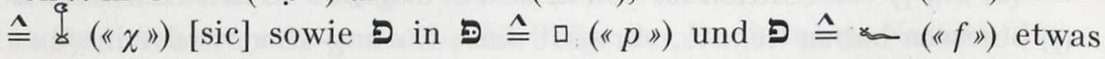
gestreckt wird $\left({ }^{2}\right)$. Entbehrlich ist dafür die Differenzierung von $ש(s, s)$

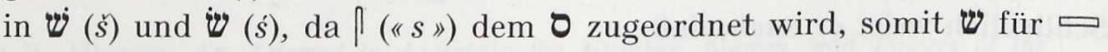
(Var. Alphabet ebensowenig erlangt wie das Champollionsche hebräische.

(1) H. Brugsch, Geographische Inschriften, Berlin, 1857-1860, Bd. I, S. 5-15; vgl. auch id., Die Ägyptologie, Leipzig, 1891, S. 42f. (zur Sache), sowie S. 42 und S. 127 (zum wissenschaftsgeschichtlichen Aspekt).

(2) Brugsch, Geographische Inschriften, S. 15; id., Die Ägyptologie, S. $125 f$. 
Angewandt hat dagegen ein modifiziertes hebräisches Alphabet Samuel Birch. Ihm ging es hierbei jedoch anders als den genannten Hincks und Brugsch nicht primär um Lautwertbestimmungen anhand des Semitischen, sondern um die Anordnung lexikalischer Entsprechungen, d.h. primär um eine Anordnungsvorschrift. Er benutzt das Alphabet in einem "Complete Comparison of the Hitherto Known Old and New Egyptian Words with the Semitic » betitelten Anhang zur englischen Ausgabe von Bunsens "Ägyptens Stellung in der Weltgeschichte » $\left(\right.$ Bd. V : 1867) $\left.{ }^{1}\right)$. Er spaltet dabei, wie dies seit Champollion üblich war, die Vokale ab und stellt sie in lateinisch-alphabetischer Folge den Konsonanten voran (a, e, i, o (Omikron), w o (Omega), u). Die Konsonantenfolge ist dagegen die des hebräischen Alphabets (b, h, f ( $q$, heute

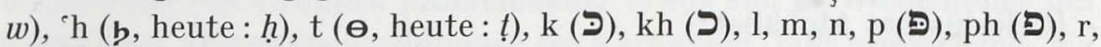
s (ש, heute: $s)$, s (ש, heute : š), t), dem jedoch als «Zusatzbuchstaben» zwei koptische «Zusatzbuchstaben » angehängt sind $(\mathrm{k}(\boldsymbol{\lambda}), \mathrm{z}(\boldsymbol{\sigma}))$.

\section{$\S$ 4. Die lautphysiologische Klassifizierung der hieroglyphischen Laut- werte}

In Christian Carl Josias Bunsens 5-bändigem Werk « ̈̈gyptens Stelle in der Weltgeschichte », das 1844 in der deutschen Originalausgabe zu erscheinen begann, findet sich im ersten Band die folgende Klassifizierung der ägyptischen Laute (in Klammern, falls abweichend, die entsprechenden heutigen Transkriptionszeichen) $\left({ }^{2}\right)$ :

-4 « reine Haucher»: $a\left(3, i / j,{ }^{c}\right), u(w), i(y$, auch $¥ i), h(h, h)$

-3 "flüssige Laute» : $m, n, r$

-2 «Zischlaute»: $s, \boldsymbol{x} / \operatorname{skh}\left(s^{\prime}\right)$

- 5 "stumme [Laute]», im einzelnen :

- 3 "Lippenl[aute]»: ‘ $b$ (frz. $v$, heute : $b), f, p$

-2 «Gaumenl[aute] : $\quad k(k / q, k, g), \chi / \operatorname{ch}(\underline{h})$

- 1 «Zungenl[aut]»: $t$

(1) C. C. J. Bunsen, Egypt's Place in Universal History, London, 1848-1867, Bd. V (1867), S. 743-773 (die tabellarische Aufstellung des Alphabets S. 745f. ist nicht ganz kongruent mit dem in der folgenden lexikalischen Liste angewandten; letzteres unten angeführt).

(2) C. C. J. Bunsen, Ägyptens Stelle in der Weltgeschichte ..., Hamburg, 1844-1857, Bd. I, S. 325 . 
Bei den Lauten selbst handelt es sich um nichts anderes als um die 15 Laute, die Richard Lepsius bereits 1837 bei der Konsolidierung des Champollionschen Erbes festgestellt hatte (s. oben $\S 2.3$ ).

\section{Anmerkung 1:}

Bei der konkreten Vorführung der Phonogramme («Lautbilder») wendet Bunsen ein Alphabet an, das eine Kreuzung zwischen lautphysiologischer Klassifikation und koptischem Alphabet darstellen könnte, letzteres zerlegt in griechisches Alphabet und koptische "Zusatzbuchstaben" (in Klammern, falls abweichend, die entsprechenden heutigen Transkriptionszeichen) (1) :

- [Griechisches Alphabet:]

- ["reine Haucher», Teil $1:] \quad a\left(3, i / j,{ }^{c}\right), u(w), i(y$, auch " $i)$

- [Konsonanten in quasi-alphabetischer Folge :]

- [«stumme (!) Lippenlaute»:] $b, f, p$

- [«flüssige Laute»:] $\quad m, n, r / l$

- [«Zischlaute», Teil $1:] \quad s$

- [Zungenlaut»:] $t$

- [koptische "Zusatzbuchstaben ":]

- [ «reine Haucher», Teil $2:] \quad h(h, h)$

- [Konsonanten, alphabetisch fehlerhaft angeordnet:]

- "stumme Gaumenlaute», auch des griechischen Teils des Alphabets (!) :]

- ["Zischlaute», Teil 2:]

$k(k / q, k, g), \chi / c h(\underline{h})$

$\boldsymbol{x} / \operatorname{sch}(s)$

(Ob die hier vorgeschlagene Analyse Bunsens im Bereich der koptischen "Zusatzbuchstaben » korrekt ist, sei dahingestellt.)

Das Alphabet hat wohl keine Nachfolge gefunden, ist aber insofern von Interesse, als die hier zum ersten Mal bezeugte Mischung der Prinzipien später noch einmal und dann mit dauerhaftem Erfolg eingeführt wird, s. unten $\S 5$. Die Art der Mischung ist jedoch zu verschieden, als daß zwischen den Späteren und Bunsen ein wissenschaftsgeschichtlicher Zusammenhang angenommen werden darf. Zudem ist die Genese der späteren Mischung unverkennbar die Folge einer ganz bestimmten Konstellation von Wissenschaftlern bzw. deren Ansichten.

(1) Bunsen, Ägyptens Stelle, Bd. I, S. 672-686. 
Anmerkung 2:

Eine ähnliche Reihenfolge, in der koptisches Alphabet und lautphysiologische Klassifikation vermischt erscheinen, findet sich bei Emanuel de Rougé in seinem "Mémoire sur l'inscription du tombeau d'Ahmès " (einer Pionierarbeit, in der zum ersten Mal ein längerer Text zusammenhängend erklärt wurde) ${ }^{(1)}$. Die Abhängigkeit von Bunsen ist deutlich. Sie zeigt sich u.a. und vor allem in der Gruppierung des $h$ zusammen mit den Vokalen. Insgesamt ist seine Aufstellung stärker von der Lautphysiologie bestimmt. Grundsätzlich läßt er die Bunsen/Lepsiusschen lautphysiologischen Gruppen bestehen (auch wenn er im einzelnen nicht an deren Korrektheit glaubt) und läßt sich nur bei der Festlegung der Reihenfolge der Gruppen untereinander etwas von der Reihenfolge des koptischen Alphabets beeinflussen (in Klammern, falls abweichend, die entsprechenden heutigen Transkriptionszeichen) $\left({ }^{2}\right)$ :

- [«reine Haucher $»]\left(^{(3)}\right.$

$\mathrm{H}(h), \mathrm{A}^{0}\left(3, i / j,{ }^{\circ}\right)\left({ }^{4}\right), \mathrm{I}^{\circ}(y$, auch

$-[$ Konsonanten :]

- ["(stumme ?) Lippenlaute» :] " $i), \mathrm{OU}^{\circ}(w)$

- [ «lüssige Laute»:]

- ["Zungenlaute»:]

$\mathrm{W}(f), \mathrm{V}(b), \mathrm{P}$

$\mathrm{M}, \mathrm{N}, \mathrm{R} / \mathrm{L}$

$\mathrm{T}(t, \underline{t}, d), \mathrm{T}^{\prime}(\underline{d})\left({ }^{5}\right)$

$\mathrm{K}(k / q, k, g)$, Ch $(\underline{h})$

$\operatorname{Sch}(\tilde{s}), \mathrm{S}$

Es ist sehr wahrscheinlich, daß auch die neuerliche lautphysiologische Klassifizierung Bunsens von Lepsius stammt, da dieser Bunsen als philologischer Berater bei der Ausarbeitung seines Geschichtswerkes zur

(1) E. DE Rougé, Mémoire sur l'inscription du tombeau d'Ahmès chef des nautiers, in Mémoires de l'Académie des inscriptions et belles lettres, série 1, tome 3, 1851, S. 1 196 (= id., Euvres diverses, Bd. 2, Paris, 1908, S. 1-202; hiernach zitiert); zur Transkription S. 7-14.

(2) DE Rougé, op. cit., S. 8f.

(3) Die Vokale sind mit Kringel ( $\left.{ }^{\circ}{ }^{\sharp}\right)$ gekennzeichnet, um sie als Transkriptionszeichen für bestimmte Hieroglyphen von den vielfältigen Lautwerten abzuheben, die sich aus den Äquivalenten in griechischen und lateinischen Namen ergeben.

(4) Tatsächlich hält de Rougé $\mathrm{A}^{\circ}$ für einen Konsonanten, nämlich $\boldsymbol{\aleph}$ ('), s. DE RougÉ, op. cit., S. 12.

(5) Zur Differenzierung der «t-Laute» s. DE RougÉ, op. cit., S. 13 ; sowie id., Notice sur quelques textes hiéroglyphiques nouvellement publiés par $M$. Greene, $\S \mathrm{I}^{\mathrm{er}}$, in $L$ 'Athenaeum français 4 (1855), S. 956-961, bes. S. 960. 
Seite stand. Auf jeden Fall spinnt Lepsius später den Faden der lautphysiologischen Klassifizierung im Rahmen seines "Allgemeinen linguistischen Alphabetes» fort, mit dem er 1855 eine generelle Lösung für das Problem der "Übertragung fremder Schriftsysteme» und "bisher noch ungeschriebener Sprachen" "in europäische Buchstaben» vorlegte ${ }^{1}$ ). Die dort gegebene mehrdimensionale Klassifikation hat Lepsius praktisch gleichzeitig in einer Arbeit "Über eine hieroglyphische Inschrift am Tempel von Edfu » in eine eindimensionale Sequenz umgeformt, die sich wie folgt aufbaut (in Klammern, falls abweichend, die entsprechenden heutigen Transkriptionszeichen) $\left({ }^{2}\right)$ :

- Konsonanten

- «Explosivae oder dividuae»:

- «Fortes»:

- «Gutturalis» :

- «Dentalis»:

$k$

- «Labialis» :

- «Lenis "

- «Labialis»:

$b$

- «Nasales»:

- "Dentalis» :

$n$

- «Labialis » :

m

- "Fricativae oder continuae»:

- «Fortes»:

- «Gutturales» :

ch (h), $h$

- «Dentales» :

sch $(\tilde{s}), s$

- «Labialis » :

$f$

- Liquidae:

$r / l(r w)$

- Vokale:

$a, i, u$

(1) R. Lepsius, Das allgemeine linguistische Alphabet, Grundsätze der Übertragung fremder Schriftsysteme und bisher noch ungeschriebener Sprachen in europäische Buchstaben, Berlin, 1855 (englische Ausgabe: Standard Alphabet for Reducing Unwritten Languages and Foreign Graphic Systems to a Uniform Orthography in European Letters, London, 1855), bes. (deutsche Ausgabe) S. 27 (allgemeine Klassifikation der Konsonanten), S. 53 (Klassifikation der hieroglyphischen Laute).

(2) R. Lepsius, Über eine hieroglyphische Inschrift, S. 112-114 (mit Modifikationen wiederholt in ID., Königsbuch, S. 169-171); hier die ursprüngliche Anordnung von 1855 (1858 setzt Lepsius die Vokale vor die Konsonanten — was die Systematik nicht beeinträchtigt - , weniger glücklich ist die Versetzung der mehrbuchstabigen Transkriptionszeichen $\operatorname{sch} / s(\tilde{s})$ und $\operatorname{ch} / \chi(\underline{\chi})$ als "Zusatzbuchstaben " an das Ende des Alphabets). 
$\S 5$. Koptisches 15-Laute-Inventar oder Erweiterung desselben; lautphysiologische Klassifikation oder Alphabet als Anordnungsvorschrift

\section{$\S 5.1$ Überblick}

Mit dem Material der vorangehenden $\S \S 2-4$ stehen alle wesentlichen Ingredienzien bereit, aus denen das heute noch geltende ägyptologische Alphabet zusammengemischt wurde. Die Art und Weise, wie diese Mischung zustande kam, gleicht einer Tragikomödie: Richard Lepsius, der systematischste Kopf unter den Beteiligten, kam nicht mehr recht von seinem 15-Laute-Inventar von 1837 los ; er interessierte sich für dessen lauiphysiologische Konstruktion und, in Zusammenhang damit, für die Frage der Transkription der einzelnen Laute, nicht aber für das praktische Problem eines Alphabets als Anordungsvorschrift. Andere, namentlich Edward Hincks und Heinrich Brugsch, sahen die Notwendigkeit der Erweiterung des 15-Laute-Inventars, oder, namentlich Heinrich Brugsch und Samuel Birch (zu letzterem s. unten $\S 6$ ), standen vor dem Problem, für lexikographische Zwecke ein Alphabet als Anordnungsvorschrift festzulegen, ohne Systematiker genug zu sein, um die hieraus resultierenden Probleme einer stringenten Lösung zuzuführen. Was als Ergebnis heute vorliegt, ist eine kuriose Mischung aus Lepsiusscher lautphysiologischer Klassifikation, koptischem Alphabet und barer Willkür.

\section{$\S 5.2$ Quantitätsangabe und untergesetzter Punkt als Diakritika}

Durch die Untersuchungen von Edward Hincks und Heinrich Brugsch (vgl. zu diesen oben §3) sowie des ergänzend zu nennenden Emanuel de Rougé war es offenkundig geworden, daß manche Zeichen, die im Lepsiusschen 15-Laute-Alphabet als Homophone galten, in älterer Zeit nicht miteinander austauschbar waren, z.B. die mit a transkribierten Zeichen $(3), \triangle(i / j)$ und $\left.-{ }^{c}\right)$ oder die " $k$-Laute" wie $\Delta(k / q)$ und $\square$ $(k)$, die "t-Laute" wie $\bullet(t)$ und $\leftrightharpoons(d$, d.i. $t)$ oder die " $h$-Laute" wie $\square$ (h) und $\&(h)\left({ }^{1}\right)$.

Lepsius ersann einen Ausweg, den er 1863 in der 2. englischen Auflage seines "Allgemeinen linguistischen Alphabets» («Standard Alphabet»)

(1) Hincks, op. cit.; E. De Rougé, Mémoire sur l'inscription du tombeau d'Ahmès, bes. S. 7-14 (erkennt - hier allein von Interesse - die Nicht-Identität von $=($ (" $t)$ und $\left.\\left(" t^{\prime} \|\right)\right)$; Brugsch, Geographische Inschriften. 
vorlegte $\left(^{1}\right)$ : Es handle sich nicht um unterschiedliche Artikulationen, vielmehr - bei den Vokalen — um unterschiedliche Länge bzw. - bei den Konsonanten - um unterschiedliche Neigung, sich mit folgenden Vokalqualitäten $\mathrm{zu}$ verbinden, $a$ einerseits und $i / u$ andererseits. Z.B. galt ihm bei den "a-Lauten" (3) als kennzeichenlos, $A_{(i / j)}$ als kurz

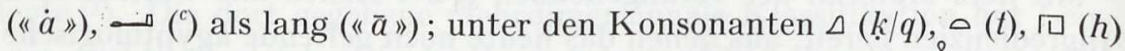
als zu $a$ tendierend ( $k a », " t a », " h a »), \smile(k), \smile(d$, d.i. $t), \quad$ \& $(h)$ als zu $i$ oder $u$ tendierend ( $k i / u », " t i / u », " h i / u »)$. Möglicherweise standen bei der Erklärung der Konsonanten die Buchstabennamen von Hincks Pate (s. oben $\S 3$ ), auch wenn die Lepsiusschen Folgevokale nur teilweise mit dem durch die Hincksschen "expletive characters" gegebenen Vokal übereinstimmen.

So weit könnte man den Lepsiusschen Lösungsvorschlag als unzutreffend einfach vergessen. Nun hat aber Lepsius zur Kennzeichnung der Vokalquantität Kürzepunkt und Längenstrich sowie zur Kennzeichnung der $i / u$-Tendenz der Konsonanten einen untergesetzten Punkt als (fakultatives) diakritisches Zeichen eingeführt, und diese Kennzeichnungen sind diejenigen, die später von anderen benutzt wurden und teilweise - die untergesetzten Punkte - bis zum heutigen Tag benutzt werden. Wenn Lepsius $\leftrightharpoons$ als « $t$ » notiert (im Gegensatz etwa zu $\bullet ~ « t »)$, dann meint er $i / u$-haltiges " $t$ » und nicht "emphatisches $t$ », als welches man « $t$ » heutzutage auffassen würde (im Lepsiusschen "Allgemeinen linguistischen » bzw. "Standard»-Alphabet wird die Emphase der semitischen Sprachen durch einen untergesetzten Strich bezeichnet, z.B. "emphatisches $t »$ als « $\underline{t} »)$.

\section{$\S 5.3$ Die Diskussion in der ZÄS 1864 bis $186 \%$ und das "Hieroglyphisch- demotische Wörterbuch» Heinrich Brugschs von $1867 \mathrm{ff}$.}

Eine lebhafte Diskussion zur Transkriptions-Frage entfaltete sich in den Jahren ab 1864 in der ein Jahr zuvor von Heinrich Brugsch begründeten "Zeitschrift für ägyptische Sprach- und Alterthumskunde » oder, wie sie seit Übernahme der Redaktion durch Richard Lepsius im Oktober 1864 hieß, "Zeitschrift für ägyptische Sprache und Alterthumskunde». Wichtig sind insbesondere die Beiträge von Heinrich Brugsch, Emanuel de Rougé und Richard Lepsius. Es ging hier um die Normie-

(1) Lepsius, Standard Alphabet, 2. Aufl., London-Berlin, 1863, S. 193-199. 


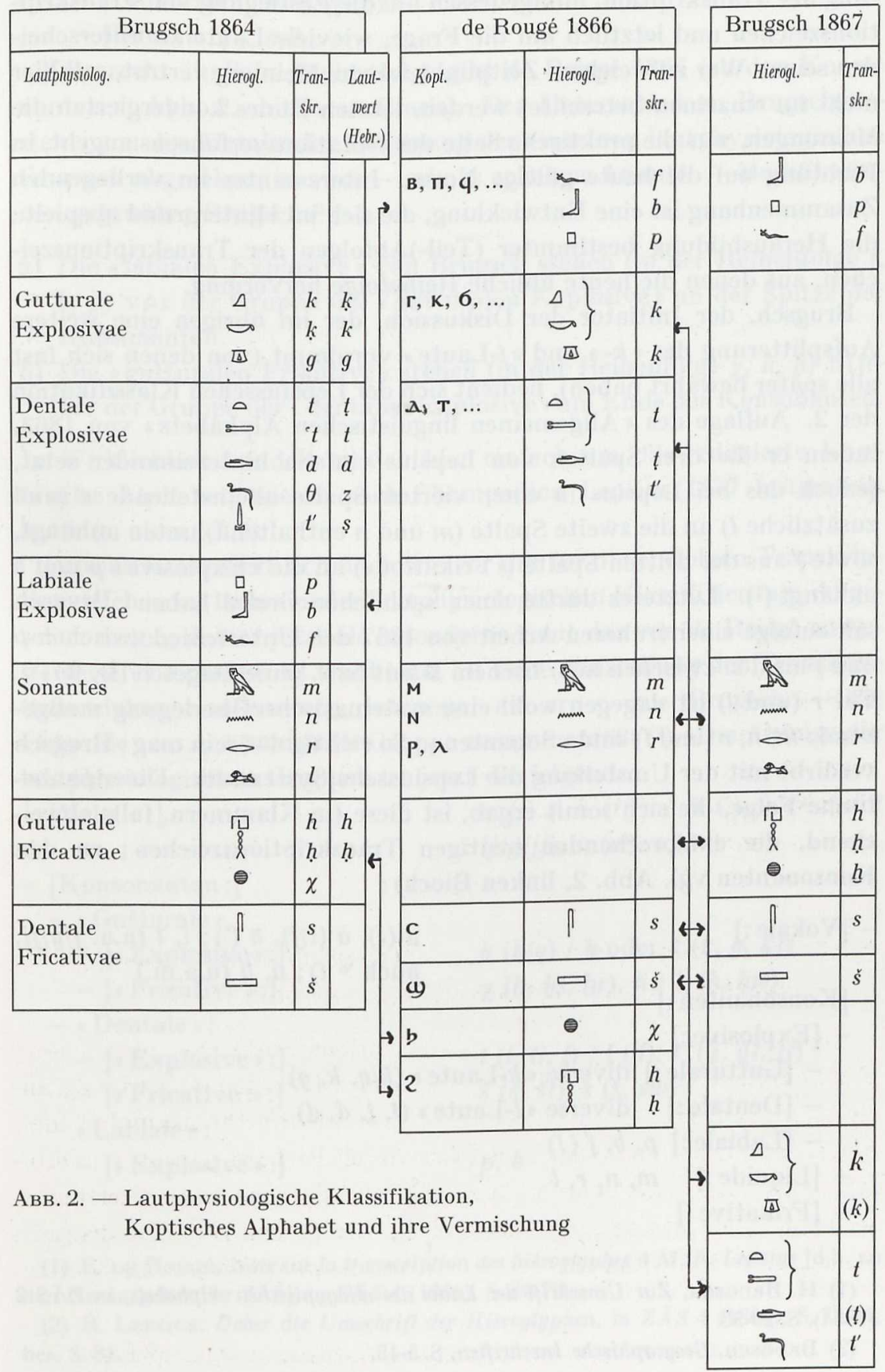


rung der Transkription, infolgedessen um die Festlegung von Transkriptionszeichen und letztlich um die Frage, wieviele Laute zu unterscheiden seien. Wer zu welchem Zeitpunkt welche Meinung vertrat, soll hier nicht im einzelnen betrachtet werden, letzten Endes konvergierten die Meinungen, was die praktische Seite des Notationsverfahrens angeht, in Richtung auf die heute gültige Norm. Interessanter im vorliegenden Zusammenhang ist eine Entwicklung, die sich im Hintergrund abspielt: die Herausbildung bestimmter (Teil-)Abfolgen der Transkriptionszeichen, aus denen die heute übliche Reihefolge hervorging.

Brugsch, der Initiator der Diskussion, der im übrigen eine weitere Aufsplitterung der " $k$-» und «t-Laute» vornimmt (von denen sich fast alle später bewährt haben), bedient sich der Lepsiusschen Klassifikation der 2. Auflage des "Allgemeinen linguistischen Alphabets » von 1863, indem er die drei Spalten von Lepsius einfach hintereinander setzt, jedoch das bei Lepsius in einer vierten Spalte alleinstehende $r$ (und zusätzliche $l$ ) an die zweite Spalte ( $m$ und $n$ enthaltend) unten anhängt, sowie $f$ aus der dritten Spalte ("Frikative») an die "Explosive" $p$ und $b$ anhängt $\left.{ }^{1}\right)$. Letzteres dürfte einen sachlichen Grund haben: Brugsch sah zufolge einer früheren Arbeit von 1857 den Unterschied zwischen $p$ und $f$ als den zwischen hebräischem $₫$ mit bzw. ohne Dagesch $(ฐ, \Xi)\left({ }^{2}\right)$. Für $r$ (und $l$ ) ist dagegen wohl eine systematische Überlegung maßgebend : $m, n, r$ (und $l$ ) sind "Sonanten". So richtig das sein mag: Brugsch verdirbt mit der Umstellung die Lepsiussche Systematik. Die alphabetische Folge, die sich somit ergab, ist diese (in Klammern, falls abweichend, die entsprechenden heutigen Transkriptionszeichen; zu den Konsonanten vgl. Abb. 2, linken Block):

- [Vokale :]

$a(3), \dot{a}(i / j), \bar{a}\left({ }^{(}\right) ; i, \bar{\imath}$ (u.a. $j / y / j j$,

$-[$ Konsonanten :] auch " $i$ ); $u, \bar{u}$ (u.a.m.)

- [Explosive :]

- [Gutturale :] diverse " $k$-Laute» $(k / q, k, g)$

- [Dentale:] diverse «t-Laute» $(t, \underline{t}, d, \underline{d})$

- [Labiale:] $p, b, f(!)$

- [Liquide:] $m, n, r, l$

- [Frikative:]

(1) H. Brugsch, Zur Umschrift der Laute des altägyptischen Alphabets, in ZÄS 2 (1864), S. 29-33.

(2) Brugsch, Geographische Inschriften, S. 5-15. 
- [Gutturale :] $h, \underline{h}, \chi(\underline{h})$

- [Dentale:] s, š

Eine recht ähnliche Reihenfolge gibt de Rougé 1866 (zu den Konsonanten s. Abb. 2, mittleren Block). Der Unterschied zu Brugsch besteht, von den Details der Besetzung der einzelnen lautphysiologischen Gruppen abgesehen, in zwei — wie es scheinen könnte - Modifikationen gegenüber Brugsch $\left({ }^{1}\right)$ :

a) Die "labialen Explosive" von Brugsch stehen (in der Reihenfolge $f$, $b, p)$ vor der Gruppe der "gutturalen Explosive» an der Spitze der Konsonanten.

b) Die "gutturalen Frikative» stehen (in der Reihenfolge $\chi, h, h$ ) hinter der Gruppe der «dentalen Explosive» am Ende der Konsonanten.

In Wirklichkeit handelt es sich hier einfach um das reduzierte koptische Alphabet, wie es nach Champollion Lepsius 1837 festgestellt hatte (s. oben $\S 2.3$ ).

Lepsius interessiert sich nach wie vor nur für die einzelnen Transkriptionszeichen im Rahmen der lautphysiologischen Klassifizierung. Abgesehen davon, daß er jetzt als besonderen Laut das von de Rougé ausgesonderte " $t$ '» (heute : $\underline{d}$ ) anerkennt, ist seine Aufstellung der Transkriptionszeichen insofern von Interesse, als er — wie Brugsch - die "Liquide» $m, n, r$ (und $l$ ) als Gruppe zusammenfaßt $\left({ }^{2}\right)$. Die Abfolge der lautphysiologischen Gruppen ist jetzt die folgende :

- [Vokale :]

$-[$ Konsonanten :]

- «Gutturale »

- [«Explosive» :]

- [ Fricative» :]

- «Dentale»:

- [«Explosive» :]

$-[$ Fricative :»:]

- "Labiale" :

- [«Explosive»:] $\left.a(3) / \dot{a}(i / j) / \bar{a}()^{\circ}\right)$, i (" $\ddot{i} / \bar{\imath}$ $(j / y / j j), u(w), o(w 3)$

$k(k / q) / k$ oder $k(k, g, k 3)$

$\chi(\underline{h}, \underline{h} 3, \underline{h} r), h / h(h, h w)$

$t(t, t i, \underline{t}) / t(d), t^{\prime}\left(\underline{d}, \underline{d}_{3}, \underline{t} 3\right)$

$\check{s}\left(\check{s}^{2}, \check{s}_{3}\right), s(s, s w)$

$p, b$

(1) E. DE Rougé; Note sur la transcription des hiéroglyphes à $M$. R. Lepsius [d.h. an den Herausgeber der ZÄS], in ZÄS 4 (1866), S. 69-73.

(2) R. Lepsius, Ueber die Umschrift der Hieroglyphen, in Z̈̈S 4 (1866), S. 73-81, bes. S. 81 . 


\section{[ Fricative»:] \\ - "Liquide» :}

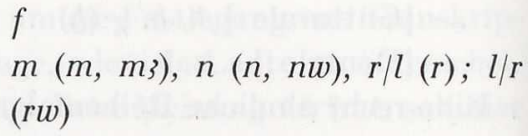

Schließlich Brugsch : In seinem seit 1867 erschienenen Monumentalwerk eines "Hieroglyphisch-Demotischen Wörterbuchs» $\left(^{(}\right)$scheint er zunächst aus de Rougés Anordnung von 1866 und seiner eigenen von 1864 einen Kompromiß geschlossen zu haben ( $\mathrm{zu}$ den Konsonanten s. Abb. 2, rechten Block). Im Falle a) folgt er de Rougé und damit dem koptischen Alphabet ("labiale Explosive» an der Spitze der Konsonanten), im Falle b) dagegen sich selbst und damit, wie im Prinzip generell, der lautphysiologischen Klassifikation von Lepsius («gutturale Fricative» vor den «dentalen Fricativen»). Völlig überraschend dagegen kommt eine Neuerung: Brugsch setzt die beiden Gruppen der "gutturalen" und "dentalen» "Explosive» an das Ende des Alphabets hinter die "Frikative» und reißt damit die "Explosive» auseinander ( Labiale» an der Spitze der Konsonanten, "Gutturale» und «Dentale» am Ende). Die einzige rationale Erklärung für diese merkwürdige Verschiebung, die mir einfällt, ist diese : Brugsch könnte mit der Verschiebung der "Gutturale" und "Dentale» eine Entscheidung in der besonders schwierigen Frage des genauen Ansatzes der Einzel-Laute in der Gruppe der "Gutturale» und "Dentale» - jedenfalls was den Anlaut angeht - vor sich her geschoben haben. Ob man jedoch überhaupt nach einer rationalen Erklärung suchen darf, ist zweifelhaft: Brugsch verwendet zu gleicher Zeit, in der er in $\operatorname{der} Z \ddot{A} S$-Diskussion sein Alphabet an die Lepsiussche lautphysiologische Klassifizierung anlehnt, an anderer Stelle ein adaptiertes koptisches Alphabet (s. oben §2.3), dessen Konstruktions-Prinzip herauszufinden ich dem Leser überlasse $\left(^{2}\right)$ :

[Vokale:] $\quad \dot{a}, \bar{a}, a, \bar{\imath}, u$

[Konsonanten:] $b, p, f, m, n, r, l, s, t, \grave{t}, t, \theta, h, h, \chi, k, s$.

$\mathrm{Zu}$ letzterem Alphabet vgl. dasjenige von Brugschs «Grammaire démotique...» von 1855 (S. 14), dessen wissenschaftsgeschichtliche Einordnung nachzuholen wäre.

(1) H. Brugsch, Hieroglyphisch-demotisches Wörterbuch, 7 Bde., Leipzig, 1867-82, bes. Bd. I (1867), S. Vf. (für die mit Bd. V (1880) beginnenden Ergänzungen gilt ein entsprechend dem Fortgang der Wissenschaft aktualisiertes Alphabet, s. Bd. V, S. 1f.).

(2) H. Brugsch, A. Rhind's zwei bilingue Papyri ..., Leipzig, 1865, S. 33-49. 
Und überhaupt: Brugsch "est d'une inexactitude révoltante», von einer himmelschreienden Ungenauigkeit, wie ich das Zitat aus einem Brief Peter Le Page Renoufs ins Deutsche übersetzen würde $\left(^{1}\right)$.

Sei dem, wie ihm wolle: In Brugschs Wörterbuch liegt in großen Zügen die Reihenfolge vor, die wir auch heute noch befolgen.

\section{$\S 6 . \quad$ Koptische Lautwerte in der Reihenfolge des lateinischen Alphabets}

Gleichzeitig mit Heinrich Brugsch sah sich Samuel Birch — der für die Zwecke des ägyptisch-semitischen Sprachvergleichs das hebräische Alphabet gewählt hatte (s. oben §3) — vor die Aufgabe gestellt, den ägyptischen Wortschatz in geordneter Folge darzustellen, als er in einem Anhang (!) zum 1867 erschienen Band V der englischen Übersetzung von Bunsens "Ägyptens Stelle in der Weltgeschichte" ein umfassendes ägyptisches Wörterbuch vorlegte $\left(^{2}\right)$. Seine Lösung der Alphabet-Frage sind koptische Lautwerte in der Folge des lateinischen Alphabets, wobei allerdings Laute, die durch eine Folge von zwei lateinischen Buchstaben dargestellt sind, als «Zusatzbuchstaben " am Ende des Alphabets stehen $\left({ }^{3}\right)$. Das Alphabet lautet (in Klammern, falls abweichend, die entsprechenden heutigen Transkriptionszeichen) :

$a(\hat{i} / j), \hat{a}(\mathcal{C}), \grave{a}(3), b, f, h(h, h), i(j / y / j j), k(k / q, k, g), m, n, p, r, s, t(t, \underline{t}, d$, d), $u(w), k h(\underline{h}), \operatorname{sh}(\dot{s})$.

\section{Anmerkung :}

In seiner ebenfalls als Anhang (!) zu Bunsens Werk erschienen ägyptischen Grammatik findet sich zusätzlich der Buchstabe « $g »(\underline{d}, \underline{t} 3, \underline{d} 3)\left({ }^{4}\right)$.

$\mathrm{Zu}$ einer späteren Anwendung der lateinischen Alphabet-Folge s. unten $\S 7.1$

(1) S. F. Chabas, Euvres diverses, Bd. 1, S. LX, Anm. 1 (Brief vom 16.8.1866, Originalsprache nicht ersichtlich).

(2) S. Birch, Dictionary of Hieroglyphics, in C. C. J. Bunsen, Egypt's Place, Bd. V, S. 335-586.

(3) Zu diesen "Zusatzbuchstaben" vgl. schon LePsius, Königsbuch.

(4) Birch, in Bunsen, Egypt's Place, Bd. V, S. 602-617, bes. S. 603. 


\section{$\S$ 7. Detail-Ausgestaltung}

\section{$\S 7.1$ Die Konvention von 1874}

Um den durch anhaltende Versuche zur Lautwertbestimmung bedingten Schwankungen in der Wahl der Transkriptionszeichen ein Ende zu bereiten, veranlaßte Richard Lepsius auf dem Orientalisten-Kongreß, der 1874 in London stattfand, die ägyptologischen Mitglieder der "Hamitischen Section", die Frage einer verbindlichen Transkriptionskonvention zu beraten $\left({ }^{1}\right)$. Damals waren die Usancen schon so eingefahren, daß man von der Darstellung einzelner Konsonanten als Vokale nicht mehr absehen mochte, obwohl man sich mehr oder minder darüber einig war, daß weder $\ell(i / j)$ noch - $\left.{ }^{c}\right)$ " $a$-Laute» waren, son-

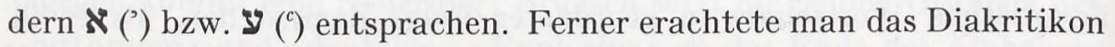
« untergesetzter Punkt» als rein konventionelles Zeichen ohne jeden Hinweis auf die lautphysiologische Realität. In anderen Fällen, in denen die Transkriptionsweise weniger fest war, versuchte man schon, lautphysiologischen Gegebenheiten Rechnung zu tragen und entsprechende Transkriptionszeichen (aus dem Lepsiusschen Allgemeinen linguistischen Alphabet) auszuwählen. Was die Reihenfolge der Transkriptionszeichen im Alphabet angeht, scheint kein Beschluß gefaßt worden zu sein. In seinem Bericht wählt Lepsius eine in seinem Sinne systematische Folge, ohne damit freilich den durch Brugschs Wörterbuch eingeführten Pervertierungen etwas anhaben zu können.

Die Transkriptionszeichen, auf die sich die Herren "Dr. S. Birch, in dessen Hause die Versammlung war, Prof. H. Brugseh-Bey aus Cairo, Prof. G. Ebers aus Leipzig, Prof. A. Eisenlohr aus Heidelberg, Prof. J. Lieblein aus Christiana, Mr. Le Page Renouf aus London, Hr. L. Stern aus Berlin und der Unterzeichnete [Prof. R. Lepsius aus Berlin]" einigten, ist - in Lepsius Reihenfolge (in Klammern die heutigen Äquivalente) - :

- [Vokale :] $a(3), \dot{a}(\hat{\imath} / j), \bar{a}\left({ }^{c}\right), i(" i), \bar{\imath}(j / y / j j)$,

- [Konsonanten :] $u(w)$

- [Gutturale :] - [Explosive :] $k, k(g), q(k / q)$

(1) R. Lepsius, Vom internationalen Orientalisten-Congre $\beta$ in London, in $Z \ddot{A} S 13$ (1875), S. 1-5, bes. S. 1 f. 
- [Frikative :]

- [Dentale:]

- [Explosive:] [Frikative :]

- [Labiale :]

- [Explosive:]

- [Frikativa:]

- [Sonanten :] $\chi(\underline{h}), h, \underline{h}$

$t, \underline{t}(d), t^{\prime}(\underline{d}), \theta(\underline{t})$

$s, s$

$p, b$

$f$

$r, l(r w), m, n$

Ludwig Stern, der an der Londoner Konferenz teilgenommen hatte, bildete aus dem Transkriptionszeichen eine lateinisch-alphabetische Folge im "Glossarium hieroglyphicum», das er zu G. Ebers' 1875 erschienener Textausgabe des Papyrus Ebers beisteuerte. Griechisches $\chi$ ist an der Stelle von lateinisch $x$ eingeordnet, während $\theta$ unter den $t$ Lauten steht. Das Alphabet lautet (in Klammern, falls abweichend, die entsprechenden heutigen Transkriptionszeichen) :

$a(3), \dot{a}(\vec{i} / j), \vec{a}\left(^{c}\right), b, f, h, h, i\left({ }^{\prime} i\right), \bar{i}(j / y / j j), k, k(g), l, m, n, p, q, r, s, \grave{s}$, $t, \theta(\underline{t}), \underline{t}(d), t^{\prime}(\underline{d}), u(w), \chi(\underline{h})$

Sterns Anordnung scheint ebensowenig wie die von Birch (s. oben $\S 6$ ) Gefolgschaft gefunden zu haben.

\section{$\S 7.2$ Semitisierung und der Oktroi von 1889}

Ein letztes Mal mit durchschlagendem Erfolg — jedenfalls auf lange Sicht - wurde 1889 die Norm der Transkription geändert. Kaum war Adolf Erman in die Herausgeberschaft der «Zeitschrift für ägyptische Sprache» eingetreten, als sich "die Redaktion» auf S. 1 in dem Sinne verlautbarte, daß die 1874 eingeführte Umschreibung (s. oben §7.1) nicht mehr dem Stand der Wissenschaft entspräche und sich «die Redaktion", um der drohenden Verwirrung wenigstens innerhalb der «Zeitschrift» Einhalt zu tun, dazu entschlossen habe, eine bestimmte Umschreibung durchzuführen (im Klartext : den Fachkollegen aufzuerlegen) $\left(^{1}\right)$. Praktisch ging es

a) um die Durchsetzung der Auffassung der einstigen Vokale als Konsonanten,

b) um die Ersetzung der griechischen Zeichen $(\chi, \theta)$ durch lateinische $(\underline{h}, \underline{t})$ und

(1) [A. Erman], Zur Umschreibung der Hieroglyphen, in Z̈̈S 27 (1889), S. 1-4. 
c) um die Erzetzung von Zeichenfolgen (genauer einer einzigen : $t^{\prime}$ ) durch ein Einzelzeichen $(\underline{d})$.

Der Sinn der Übung war eine Umgestaltung der Transkription im Sinne der zeitgenössischen semitistischen Transkription, d.h. der Transkription der «Zeitschrift der Deutschen Morgenländischen Gesellschaft», soweit dies möglich war bzw. schien. Hierzu gleich mehr. Zunächst ein paar Worte zur Reihenfolge der Transkriptionszeichen und zur Einzelgestalt derselben.

Auch wenn sich «die Redaktion" nicht ausdrücklich zur Reihenfolge der Zeichen im Alphabet äußert, so ersieht man an der späteren Verwendung der hier gewählten Reihenfolge der Transkriptionszeichen in den späteren Werken der "Berliner Schule», daß sie als Alphabet verstanden wurde. Diese Reihenfolge entspricht im wesentlichen dem unvernünftigen Alphabet, das Brugsch für sein Wörterbuch festgelegt hatte ( $y$ und " $\ddot{\imath}$ stehen bei Brugsch an anderer Stelle; einzelne Zeichen von Brugsch wurden ersatzlos gestrichen : $\left.\bar{u}\left(w_{3}\right), l\right)$.

Nur noch in Kleinigkeiten unterscheidet sich das Alphabet von unserem heutigen : A $(j / y / j j)$ und " $(i)$ stehen, anders als bei Brugsch und anders als bei Erman hinter $\hat{\imath}$ (sofern man ihnen überhaupt einen eigenen Platz zuweist); $s$ war damals noch nicht in $s / z$ und $\dot{s} / s$ zerlegt worden (und wird auch heute nicht in jedem Fall zerlegt), $\underline{h}$ ging noch mit $\underline{h}$ zusammen. Hier die Sequenz von 1889:

$$
\text { 3, } \vec{i},{ }^{c}, w, y, \ddot{i} \text { (") }, b, p, f, m, n, r, h, \underline{h}, \underline{h}, s, \stackrel{s}{s}, \underline{k}, k, g, t, \underline{t}, d, \underline{d}
$$

Wie schlecht Erman und später auch seine Mannen, die «Berliner Schule», bei ihrer Semitisierung in Fragen der Transkription und des Alphabetes beraten waren, zeigt sich besonders gut in einem wenig später erschienenen Aufsatz Georg Steindorffs, der im Zuge der neuerlichen Normierungsbestrebungen der Deutschen Morgenländischen Gesellschaft in deren Zeitschrift die Frage des "altägyptischen Alphabets und seiner Umschreibung» in einiger Ausführlichkeit behandelte (mit dem entlastenden Verweis jedoch auf eine «demnächst erscheinende 'Ägyptische Lautlehre'" aus seiner Feder, die bis zum heutigen Tag — zum mindesten als solche - nicht erschienen ist) ${ }^{1}$ ). Besonders zwei Sachverhalte verdienen hervorgehoben $\mathrm{zu}$ werden :

- Sehr richtig sieht Steindorff (S. 728), daß Ermans Wahl von $d$ als Transkriptionszeichen für $\leftrightharpoons$ unglücklich ausgefallen ist, wenn es sich

(1) G. SteindorfF, Das altägyptische Alphabet und seine Umschreibung, in ZDMG 46 (1892), S. 709-730. 
bei $\Longrightarrow$ - wie er nicht zuerst feststellte - um einen emphatischen Laut handelt. Ebenso kann man ihm folgen, wenn er als Transkriptionszeichen für $\leftrightharpoons$ lieber $t$ wählen würde. Eine solche Wiedergabe hatte sich damals nämlich bei der Deutschen Morgenländischen Gesellschaft als Norm durchgesetzt, und es war sicher zweckmäßig, auch die ägyptologische Konvention mit der semitistischen abzustimmen. Wenn Steindorff allerdings meint, damit zur Lepsiusschen Umschreibung $t \mathbf{z u}-$ rückzukehren, so ist das nur in einem äußerlichen, von ihm kaum gemeinten Sinne richtig. Für Lepsius hatte nämlich das Diakritikon Punkt einen völlig anderen Sinn, s. oben $\S 5.2$.

- Steindorff beschreibt ausdrücklich den untergesetzten Strich in der Transkription von $\rightleftharpoons$ und ${ }^{2}$ als $\underline{t}$ und $\underline{d}$ (oder wie er letzteres lieber notieren würde: $t$ ) als Notation des spirantischen Charakters von $\circlearrowright$ und 7 . Den Strich selbst hatte Erman aus der semitistischen Transkription der Deutschen Morgenländischen Gesellschaft übernommen. Der Sache nach stammt die Auffassung der Schriftzeichen von Lepsius, der $\Longleftarrow$ durch $\theta$ wiedergab, was in seinem «Allgemeinen linguistischen Alphabet» die Wiedergabe etwa des englischen stimmlosen th ist ( 2 wird von Lepsius noch nicht als eigener Laut anerkannt). Was Steindorff ebenso wie Erman unbekannt gewesen zu sein scheint, sind die sehr viel besseren Ansätze der Älteren, vor allem die von Hincks, s. oben $\S 3$.

Einen Fortschritt bedeutet dagegen die Trennung von $-\infty(s / z)$ und $\uparrow$ $(s / s)$, die Steindorff nach einer Beobachtung Fritz Hommels $\left(^{1}\right)$ an den neuentdeckten Pyramidentexten als $s$ und $s$ - in dieser Reihenfolge an die Stelle des zuvor einzigen "s-Lautes" in das Alphabet einsetzte, desgleichen die Absonderung des als $\underline{h}$ von $\underline{h}$ und seine alphabetische Einordung hinter (über die Lautwerte der " $s$-Laute " und des $\underline{h}$ ist bis zum heutigen Tag nicht das letzte Wort gesprochen). Umgekehrt schied Steindorff $A A_{j / y / j j}$ und " $\ddot{z}$ wieder aus dem Alphabet aus. Für die älteste Zeit, an der er sich orientierte, ist dies sicherlich richtig, nicht aber für die spätere (mindestens seit dem Mittleren Reich), in der $\$ A$ $j / y / j j$ Phonemstatus erreicht, zum mindesten in Endungen, in denen ein Lautwandel $w>j / y / j j$ zu beobachten ist. Abgesehen von Schwankungen, die durch letzteren Punkt verursacht sind, und unter Außerachtlassung der Steindorffschen Vertauschung der Reihenfolge von 3 und $i$ (die

(1) F. Hommel, $\bigcap$ und $\rightarrow$ als verschiedene Laute im Altägyptischen, in ZÄS 30 (1892), S. 9-11. 
nicht rezipiert wurde) $\left(^{1}\right)$, sind damit definitiv Umfang und Reihenfolge des Alphabets festgelegt, die heute noch als Standard zu betrachten sind. Bei der Wiedergabe der einzelnen Zeichen freilich sind später Verschlimmbesserungen vorgenommen worden $(j$ statt $i, z$ statt $s, q$ statt $k$ u.a.m.). Deren Behandlung liegt allerdings außerhalb des Rahmens der gegenwärtigen Untersuchung, da Konsequenzen für die alphabetische Reihenfolge der Transkriptionszeichen nicht gezogen wurden.

Ermans hemdsärmeliges Vorgehen - Entscheidungen ohne eine hinreichende Kenntnis der Sekundärliteratur — führte durch den Erfolg seiner Schule zu einem Bruch in der Tradition der wissenschaftlichen Fragestellung. In anderen Bereichen der ägyptischen Sprachwissenschaft verhält es sich nicht viel anders. Während jedoch in fast allen anderen Bereichen durch Erman und seine Mitstreiter und Nachfolger von den Quellen her ein neues Fundament gelegt wurde und ein Rückgriff über Erman zurück heute selten mehr ergiebig ist - es sei denn, man interessiere sich für Wissenschaftsgeschichte als solche - ist dies im Bereich der Lautwertbestimmungen nicht mit der erforderlichen Gründlichkeit geschehen. So durchschlagend war aber die Wirkung der Berliner Entscheidungen, daß trotz der Nachbesserungsversuche von Leuten wie Wilhelm Czermak $\left(^{2}\right)$ oder Otto Rössler $\left(^{3}\right)$ in manchen Einzelfragen bis zum heutigen Tag nicht wieder das Niveau erreicht ist, das Edward Hincks bereits in seiner Abhandlung von 1847 erreicht hatte, und damit heute keine wesentlich bessere Basis für die Konstruktion eines Alphabets besteht als Mitte des letzten Jahrhunderts.

Aber auch die Gegner der "Berliner Schule» waren um keinen Deut besser : Z.B. hing Édouard Henri Naville (1844-1926), dem ein besonderes Verhältnis zu Lepsius nachgesagt wird $\left(^{4}\right)$, zeitlebens an der Vorstellung, die vorderen Laute im ägyptologischen Alphabet stellen Vokale dar und nicht, wie die "Berliner Schule» wollte, Konsonanten $\left(^{5}\right)$. Offensichtlich erinnerte er sich nicht mehr an den von Lepsius verfaßten

(1) Diese Reihenfolge findet sich zuerst wohl bei E. DE RougÉ, ZÄS 4 (1866), S. 70.

(2) W. Czermak, Die Laute der ägyptischen Sprache, Teil 2, Schriften der Arbeitsgemeinschaft der Ägyptologen und Afrikanisten in Wien 3, Wien, 1934 (Die Laute des Neuägyptischen, S. 191-258 des Gesamtwerks).

(3) O. Rössler, Das Ägyptische als semitische Sprache, in F. Altheim/R. Stienl, Christentum am Roten Meer, Bd. I, Berlin - New York, 1971, S. 263-326 (und andere Arbeiten dieses Verfassers).

(4) S. A. Erman, Mein Leben und mein Wirken, Leipzig, 1929, S. 170.

(5) So noch 1926, in seinem Todesjahr: E. Naville, L'écriture égyptienne, Paris, 1926, S. 117-127. 
Bericht über die Konvention von 1874, in der die Wiedergabe der betreffenden Laute als Vokale klar und deutlich als konventionell bezeichnet wird, erinnerte er sich nicht mehr an Brugsch, der die konsonantische Natur zum mindesten eines Teiles der Laute gesehen hatte, nicht zu reden von Hincks, der nie in vollem Umfang in der Ägyptologie rezipiert wurde. Bei dieser Gelegenheit noch eine «Blüte» des Rezensionswesens, die manchen heutigen Produkten in nichts nachsteht: die Besprechung zu Band 1 und 2 des Setheschen Verbums aus der Feder von Karl Piehl, in der Rezensent zwar dem Fleiß von Verfasser uneingeschränkt Tribut zollt, sich ansonsten aber genötigt sah, "wesentliche Ausstellungen $\mathrm{zu}$ machen » $\left.{ }^{1}\right)$. Diese betreffen die Nicht-Berücksichtigung des Demotischen und - das ist das Hauptanliegen der Rezension - die konsonantische Auffassung der vorderen Zeichen des ägyptologischen Transkriptionsalphabets. Rezensent rät Verfasser zusammenfassend, "sich vor Druck des dritten Bandes durch das Studium des Demotischen zu einer gesunden Ansicht über die aegyptischen Vokale und deren Umschreibung zu bekehren ». Man braucht kein Bewunderer des Setheschen Denkens zu sein, um diese Stellungnahme als absurd zu empfinden. - In der Frage der alphabetischen Reihenfolge waren die Gegner der «Berliner Schule» genauso unkritisch wie diese; man hielt sich an die von Brugsch zusammengeschusterte Sequenz $\left({ }^{2}\right)$.

Man sieht: Nicht erst heute wird die Fachliteratur nur oberflächlich oder gar nicht gelesen.

Was Erman (und die späteren Berliner) von der lautlichen Realität zu wissen glaubten und was sie als bloße Notationskonvention betrachteten, ist nicht ganz leicht zu ermitteln. Ermans abschließende Stellungnahme von 1896 betont sehr stark den rein konventionellen Charakter der Transkription $\left({ }^{3}\right)$. Aber es ist derselbe Erman, der wenige Jahre zuvor die besteingeführte Konvention, die von 1874, umgeworfen hatte, nur um sein Konzept der Vokallosigkeit der Schrift und des generell semitischen Charakters der ägyptischen Sprache zur Anschauung zu bringen. Also war die Transkription für ihn doch mehr als bloße Konvention. Ich habe den Eindruck, daß Erman in den neunziger Jahren taktisch abwiegelt: Nachdem er in den ihm wesentlichen Punkten für seine Zeitschrift eine neue Transkription durchgesetzt hatte, versuchte er, Nicht-Überzeugten deren Anwendung mit der Überlegung schmack-

(1) [Karl Piehl], Sphinx 3 (1900), S. 228-232.

(2) S. z.B. H. Gauthier, Dictionnaire des noms géographiques ..., Kairo, 1925-31, Bd. I, S. VI.

(3) A. Erman, Die Umschreibung des Ägyptischen, in ZÄS 34 (1896), S. 51-62. 
haft zu machen, jede Transkription sei konventionell, es käme also nicht darauf an, welche man anwendet - warum dann nicht die seine? Es machte sich dann gut, die Punkte der Transkription herauszustellen, zu denen Erman keine dezidierte Meinung hatte und in denen sie also auch für ihn einen bloß konventionellen Charakter besaß (konkret: Unklarheiten bei den «t-» und « $k$-Lauten »). Generell glaubte Erman schon an die "Richtigkeit" seiner Transkription. Diesen Eindruck scheint mir Hermann Ranke zu bestätigen, der sich 1934 anläßlich der Besprechung von Wilhelm Czermaks "Lauten der ägyptischen Sprache » der Zeit seiner Erlernung der ägyptischen Sprache "vor mehr als 30 Jahren " erinnert: "... wenn auch Erman in der ersten Auflage seiner ägyptischen Grammatik [von 1894] bei Besprechung des 'Alphabets' wohlweislich betonte, 'unsere Umschreibung dieser Zeichen' dürfe 'nur als eine ungefähre Wiedergabe der betreffenden Laute gelten', so glaubten wir doch, im großen und ganzen auf gesichertem phonetischem Boden zu stehen $\left.{ }^{1}\right)$.

\section{§. Fazit}

Um auf die eingangs ( $§ 1$ ) aufgeworfenen Fragen zurückzukommen : Das heutige ägyptologische Transkriptionsalphabet ist ein Mixtum compositum aus unterschiedlichen Problemlösungsansätzen der Ägyptologie des 19. Jh. mit einem Residuum an Details, für die damals eine sachgemäße Lösung nicht gefunden wurde. Im einzelnen lassen sich folgende Komponenten ausmachen :

a) Kern des Alphabets ist das lautphysiologisch klassifizierte 15-LauteInventar von Richard Lepsius, dessen Systematik infolge der Auflösung in eine lineare Sequenz etwas gelitten hat, aber immer noch zu guten Teilen am heutigen Alphabet selbst ablesbar ist.

b) Die Scheidung der Vokale (bzw. der Konsonanten, die einmal für Vokale gehalten wurden) von den Konsonanten ist nicht erst eine Folge der lautphysiologischen Klassifizierung durch Lepsius, sie geht vielmehr auf Champollion zurück.

c) Die Position der Gruppe der «Labiale» zu Beginn der Konsonantenreihe ist die Folge einer Kreuzung der lautphysiologisch-strukturierten Sequenz mit dem koptischen Alphabet: Die «Labiale» stehen an der Stelle des ersten Konsonanten im koptischen Alphabet, des B (b).

(1) H. Ranke, OLZ 37 (1934), Sp. 290. 
d) Die Position der "gutturalen » und «dentalen » «Explosive " am Ende des Alphabets könnte u.U. auf einer arbeitspraktischen Entscheidung Heinrich Brugschs beruhen (andernfalls hätte sie als bare Willkür zu gelten).

e) Die Reihenfolge der Konsonanten innerhalb der lautphysiologischen Gruppen ist im wesentlichen ein Zufallsergebnis, weil zur Zeit der Festlegung der Reihenfolge die Lautwerte der einzelnen Laute innerhalb der Gruppen entweder nicht genauer bestimmbar waren oder zwar bereits näher bestimmt waren, aber denjenigen, die das Alphabet festlegten, nicht (mehr) bekannt waren, in jedem Falle sich somit einer lautphysiologischen Feinsortierung entzogen.

\section{$\S$ 9. Schlußbetrachtung: Soll man bei der Konvention bleiben?}

Das ägyptologische Transkriptionsalphabet ist das Ergebnis einer der vielen innerägyptologischen Sonderentwicklungen, die dem Ägyptologen wenig nutzen und dem Nicht-Ägyptologen den Zugang, wenn nicht gerade verbauen, so doch erschweren. So verkonstruiert, wie das Transkriptionsalphabet ist, stellt es bestimmt kein hohes Kulturgut dar, könnte also verworfen und durch ein rational konstruiertes oder ohne besondere Vorbereitungen benutzbares (wie etwa das lateinische) ersetzt werden. Dem jedoch steht ein wichtiges Argument entgegen: Eine rationale Konstruktion oder die Anlehnung an ein geläufiges Alphabet setzt voraus, daß man sich über die einzelnen Transkriptionszeichen und die damit bezeichneten Laute definitiv einigt. Dies aber ist zur Stunde nicht möglich.

Nach dem Widerstreit zwischen koptisch-orthodoxen Franzosen, den Champollion-Nachfolgern de Rougé und vor allem Chabas, dem systematischen Deutschen, Lepsius, dem lateinisch-praktischen Briten, Birch, haben der geniale Schlamper Brugsch im Verein mit dem burschikosen Erman immerhin eines bewirkt : einen festen Rahmen, innerhalb dessen man ruhig mit einzelnen Lautwerten und deren Notation experimentieren kann. Ob man nun $\hat{A}$ als $i$ verstand oder als $j$, ob man $\triangle$ als $q$ verstand oder als $k$ : Man brauchte in der Vergangenheit nicht bei jeder Uminterpretation gleich das Alphabet zu ändern. Eine Revision der alphabetischen Reihenfolge der Transkriptionszeichen sollte man dann doch wohl bis zu dem Zeitpunkt vertagen, zu dem die Lautwerte einigermaßen abschließend beurteilt und die diese bezeichnenden Transkriptionszeichen sachgerecht festgelegt werden können.

\section{Wolfgang Schenkel}

